\title{
Effects of adriamycin and candesartan on the collagen and elastin of the aorta in rats
}

\author{
Jae-Sun Uhm ${ }^{1 \dagger}$, Woo-Baek Chung ${ }^{2 \dagger}$, Jung-Sook Yoon ${ }^{3}$, Yong-Seog $\mathrm{Oh}^{2}$ and Ho-Joong Youn ${ }^{2 *}$
}

\begin{abstract}
Introduction: It has been reported that the chemotherapeutic agent, adriamycin, not only has an effect on the myocardium but also on the arteries. The aim of this study is to elucidate effects of adriamycin and an angiotensin receptor blocker, candesartan, on collagen and elastin of the aorta in rats.

Methods: Twenty four male 8-week-old Wistar-Kyoto rats were divided into four groups: control (C) group, adriamycin-treated (AD) group, candesartan-treated (CA) group, and adriamycin- and candesartan-treated (AD + CA) group. Adriamycin of $2.5 \mathrm{mg} / \mathrm{kg} / \mathrm{wk}$ was administered intraperitoneally one time per week for 6 weeks, and candesartan of $10 \mathrm{mg} / \mathrm{kg} /$ day was administered orally everyday for 6 weeks. After 6 weeks, the rats were sacrificed and the aortas were harvested. Hematoxylin-eosin staining, Verhoff's elastic, and Goldner's trichrome staining were performed for histopathologic analyses. Tunica media thickness, collagen, and elastic area fractions were measured quantitatively with a computerized digital image analyzer.
\end{abstract}

Results: Tunica media thickness in the $C A$ and $A D+C A$ groups was significantly lesser than that in the $C$ and $A D$ groups, respectively. The $A D$ and $A D+C A$ groups had a tendency of lower elastin area fraction than the $C$ and $C A$ groups, respectively. Collagen area fraction in the AD + CA group was significantly lower than that in the AD group. There were no significant differences of collagen/elastin ratio between groups.

Conclusions: These findings suggest that adriamycin has a tendency of decreasing the quantity of elastin fibers and candesartan cannot mitigate the effects of adriamycin on elastin fibers.

Keywords: Doxorubicin, Aorta, Candesartan, Collagen, Elastin

\section{Introduction}

The anthracycline chemotherapeutic agent, adriamycin, is widely used and effective in many malignancies [1]. However, adriamycin has an important adverse effect, cardiotoxicity [2]. In practice, oncologists cannot often continue with the administration of adriamycin because of adriamycin-induced cardiomyopathy, though adriamycin is effective in inducing remission of cancer.

The aorta has the same embryologic origin as the heart, and it is anatomically and hemodynamically directly connected to the heart. The heart and the aorta often share the same pathophysiology of the diseases including hypertensive cardiovascular disease. Some researchers proposed that adriamycin has adverse effects not only on the heart but also on the arteries [3-5]. However, there have been not many studies on vascular pathology by adriamycin, and changes of aorta microstructure by adriamycin have not been known in detail. In addition, it has been reported that angiotensin-converting enzyme inhibitors or angiotensin receptor blockers can alleviate the fibrotic process [6]. This was a pilot study to investigate whether adriamycin can have an effect on collagen and elastin of the aorta in rats and whether candesartan can mitigate the effects of adriamycin.

\footnotetext{
* Correspondence: younhi@catholic.ac.kr

${ }^{\dagger}$ Equal contributors

${ }^{2}$ Department of Cardiology, Catholic University of Korea College of Medicine, Seoul, Korea

Full list of author information is available at the end of the article
}

\section{Methods \\ Animals}

The study protocol was approved by the institutional review board for Animal Research of Yeouido St. Mary's Hospital (no: YEOUIDO-2012-13-01). Twenty four male 


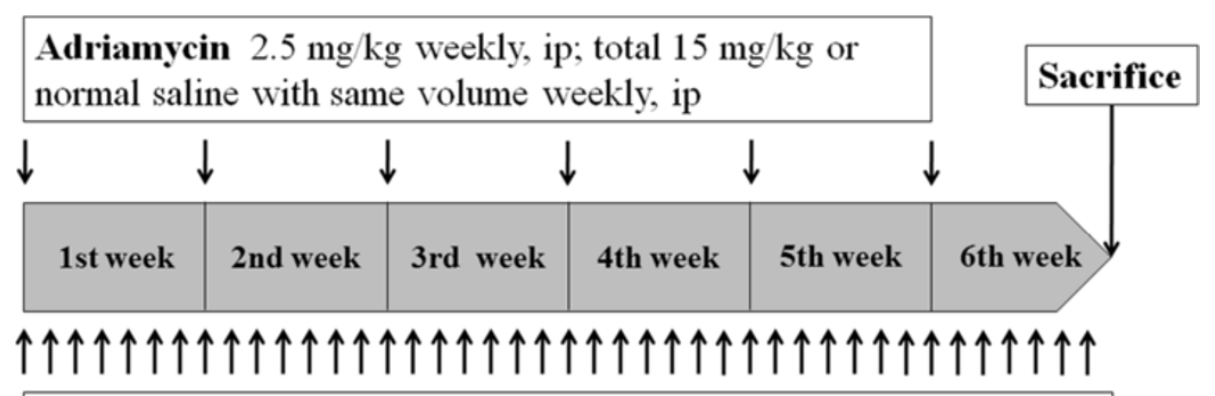

Candesartan $10 \mathrm{mg} / \mathrm{kg}$ or normal saline with same volume qd po

Figure 1 Schedule of administration of adriamycin and candesartan. ip, intraperitoneally; po, per os; qd, everyday.

8-week-old Wistar-Kyoto rats were used. The rats were kept in cages under conditions of a room temperature of $20^{\circ} \mathrm{C}$, a relative humidity of $60 \%$, and a day/night cycle of $12 \mathrm{~h}$. The rats had free access to food and water in cages. The rats were divided into four groups: control $(\mathrm{C})$, adriamycin-treated (AD), candesartan-treated (CA), adriamycin- and candesartan-treated $(\mathrm{AD}+\mathrm{CA})$. The rats were weighed at the beginning of the experiment. In the $\mathrm{AD}$ and $\mathrm{AD}+\mathrm{CA}$ groups, $2.5 \mathrm{mg} / \mathrm{kg}$ of adriamycin (Ildong Pharmaceuticals Co., Anseong, Korea) was injected intraperitoneally six times for 6 weeks (Figure 1) [7]. In the C and CA groups, normal saline of the same volume was injected by using the same method at the same time as the $\mathrm{AD}$ and $\mathrm{AD}+\mathrm{CA}$ groups. In the $\mathrm{CA}$ and $\mathrm{AD}+\mathrm{CA}$ groups, $10 \mathrm{mg} / \mathrm{kg} /$ day of candesartan (AstraZeneca, Zoetermeer, The Netherlands) was administered orally with a sonde (Figure 1 ). In the $C$ and $A D$ groups, normal saline of the same volume was administrated orally by using the same method at the same time as the CA and AD + CA groups. After 6 weeks, the rats were weighed and placed under anesthesia by zoletil (Virbac, Carros, France), the sternum was incised longitudinally, and the heart and the aorta were exposed. The rats were euthanized by injection of potassium chloride into both ventricles. After cardiac arrest, the aorta was extracted from the proximal ascending aorta to bifurcation of both iliac arteries.

\section{Staining and light microscopic examination}

After the extracted aorta was irrigated with normal saline, the descending thoracic aorta was cut into small pieces. These pieces were fixed in $10 \%$ neutral-buffered formalin solution. After the dehydration process, the aortic tissue sample was embedded into a paraffin block and cut with a microtome. Hematoxylin-eosin staining, Verhoff's elastin staining, and Goldner's trichrome staining were performed for light microscopic examination. Elastin was stained black using the Verhoff's elastin staining, and collagen was stained green using the Goldner's trichrome staining. Photos of the aorta tissue were taken from six different areas per a rat at a magnification of $\times 400$ with a light microscope (Zeiss, Jena, Germany). The photo images were quantitatively analyzed with Image-Pro Plus (MediaCybernetics, Silver Spring, MD, USA), a computerized digital image analyzer. Tunica media thickness was measured with a digital ruler and a microscopic scale in hematoxylin-eosin-stained section. The area in black was semi-automatically measured with Image-Pro Plus in Verhoff's elastin-stained sections for measurement of the elastin area. The area in green was semi-automatically measured with Image-Pro Plus in Goldner's trichromestained section for measurement of the collagen area. Elastin area fraction was calculated by dividing the elastin area by the total sampling area, and collagen area fraction was calculated by dividing the collagen area by the total

Table 1 Changes of body weight before and after administration of drugs

\begin{tabular}{llll}
\hline Group & BW1 (g) & BW2 (g) & DBW/BW1 × 100 (\%) \\
\hline C & $180.5(164-192.5)$ & $289.5(265.5-296.5)$ & $60(54-62)$ \\
AD & $186.5(186-194.5)$ & $262(247-266)$ & $40(27-43)$ \\
CA & $170.5(167-190.5)$ & $235.5(239.5-287)$ & $38(26-71)$ \\
AD + CA & $172.5(158-174.5)$ & $224(210-244.5)$ & $40(22-42)$ \\
\hline
\end{tabular}

$B W 1$ body weight before administration of drugs, $B W 2$ body weight after administration of drugs, $\triangle B W B W 2-B W 1, C$ control, $A D$ adriamycin-treated, $C A$ candesartan-treated. 
sampling area. Collagen/elastin ratio was calculated by dividing the collagen area by the elastin area.

\section{Electron microscopic examination}

The aortic tissue was fixed in $2.5 \%$ phosphate-buffered glutaraldehyde for $2 \mathrm{~h}$ and fixed in 1\% osmium tetroxide and then dehydrated in serial alcohol. The aortic tissue was embedded in araldite and sectioned. After staining with toluidine blue, the aortic tissue sections were observed under microscope for precise location to cut for ultrathin sections. The endothelial and medial areas of the aorta were observed with an electron microscope (EM, Zeiss) at magnifications of $\times 8,000$ and $\times 16,000$, respectively.

\section{Statistical analysis}

Because of nonnormal distribution of data, the results were expressed as median (interquartile range). KruskalWallis test was used for comparison of baseline characteristics of continuous variables between the four groups. For
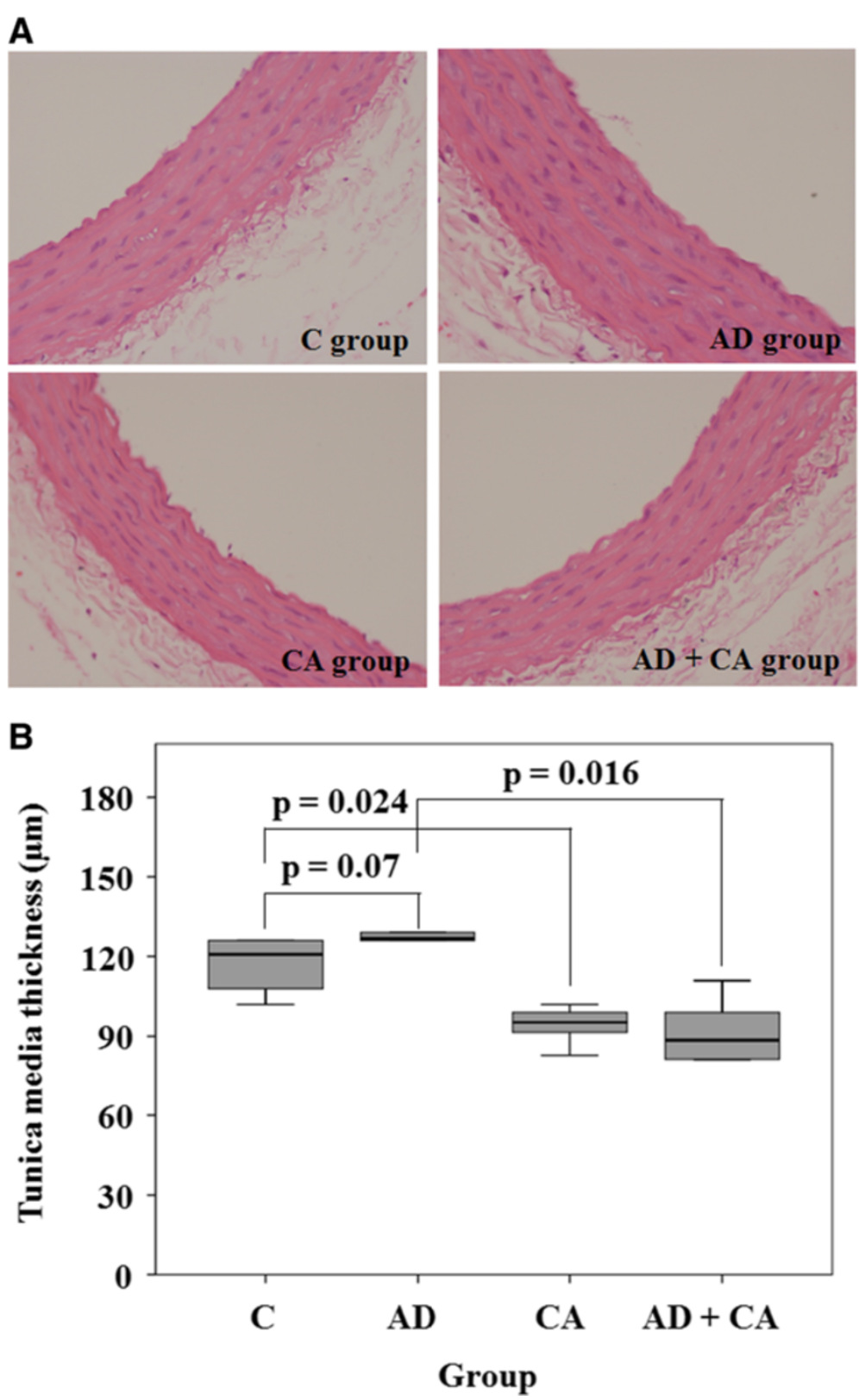

Figure 2 Hematoxylin-eosin-stained section and tunica media thickness. (A) Hematoxylin-eosin-stained section ( $x$ 400) in C group, AD group, $C A$ group, and $A D+C A$ group. (B) Tunica media thickness within the aorta in each group. C, control; AD, adriamycin-treated; $C A$, candesartan-treated; $A D+C A$, adriamycin + candesartan-treated. $p$ values were corrected by Dunnett method. 
post hoc analysis, we used Mann-Whitney $U$ test with Dunnett comparisons between two groups: [8] the C group and the $\mathrm{AD}$ group, between the $\mathrm{C}$ group and the $\mathrm{CA}$ group, between the $\mathrm{AD}$ group and the $\mathrm{AD}+\mathrm{CA}$ group, between the $\mathrm{CA}$ group and the $\mathrm{AD}+\mathrm{CA}$ group, and between the $\mathrm{C}$ group and the $\mathrm{AD}+\mathrm{CA}$ group. $\mathrm{A}$ $p$ value and Dunnett-corrected $p$ value $\left(p_{\text {Dun }}\right)<0.05$ was considered statistically significant. The data were analyzed using the IBM SPSS ver. 20.0 (IBM Co., Armonk, NY,
USA) and R Package ver. 2.0 (R Foundation for Statistical Computing, Vienna, Austria).

\section{Results}

All of the 24 rats survived throughout the study period. There were no significant differences in body weights at the beginning of the study between the groups $(p=0.270)$ (Table 1). However, the rats in the $\mathrm{AD}$ group, the CA group, and the $\mathrm{AD}+\mathrm{CA}$ group had a tendency to have
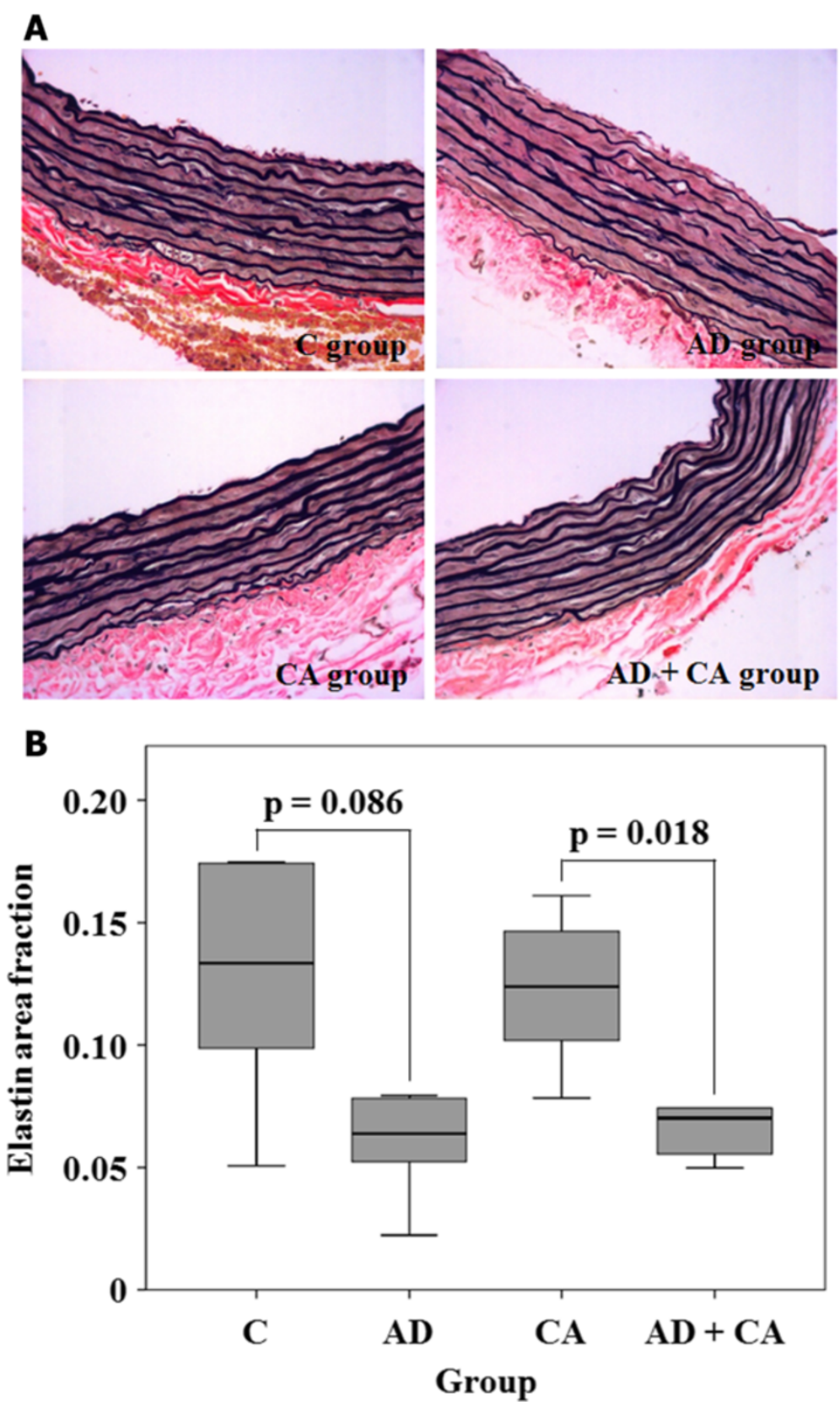

Figure 3 Verhoff's elastin stained section and elastin area fraction. (A) Verhoff's elastin-stained section $(\times 400)$ in $C$ group, AD group, CA group, and $A D+C A$ group. (B) Elastin area fraction within the aorta in each group. C, control; $A D$, adriamycin-treated; $C A$, candesartan-treated; $\mathrm{AD}+\mathrm{CA}$, adriamycin + candesartan-treated. $p$ values were corrected by Dunnett method. 
lower weight gain compared with the rats in the $C$ group, although there was no statistical significance $(p=0.223)$ (Table 1). There were no complications of intraperitoneal injection or oral administration. In hematoxylin-eosinstained section, the tunica media thickness were $121.0 \mu \mathrm{m}$ (range, 105.25 to $126.0 \mu \mathrm{m}$ ), $127.25 \mu \mathrm{m}$ (range, 121.0 to $137.75 \mu \mathrm{m}$ ), $95.25 \mu \mathrm{m}$ (range, 87.0 to $100.25 \mu \mathrm{m}$ ), and $89.0 \mu \mathrm{m}$ (range, 80.75 to $104.75 \mu \mathrm{m}$ ) in the $C$ group, the $\mathrm{AD}$ group, the $\mathrm{CA}$ group, and the $\mathrm{CA}+\mathrm{AD}$ group, respectively. Tunica media thickness in the CA group was significantly lesser than that in the $C$ group $\left(p_{\text {Dun }}=0.024\right)$. Tunica media thickness in the AD + CA group was significantly lesser than that in the $\mathrm{AD}$ group $\left(p_{\text {Dun }}=0.016\right)$ (Figure 2). However, there were no significant differences in tunica media thickness between the $\mathrm{C}$ group and the $\mathrm{AD}$ group and between the CA group and the $\mathrm{AD}+\mathrm{CA}$ group. In Verhoff's elastin-stained section, the elastin area fraction were 0.15 (range, 0.08 to 0.19 ), 0.07 (range, 0.04 to 0.09 ), 0.14 (range, 0.10 to 0.17 ), and 0.08 (range, 0.06 to 0.11 ) in the $\mathrm{C}$ group, the $\mathrm{AD}$ group, the $\mathrm{CA}$ group, and
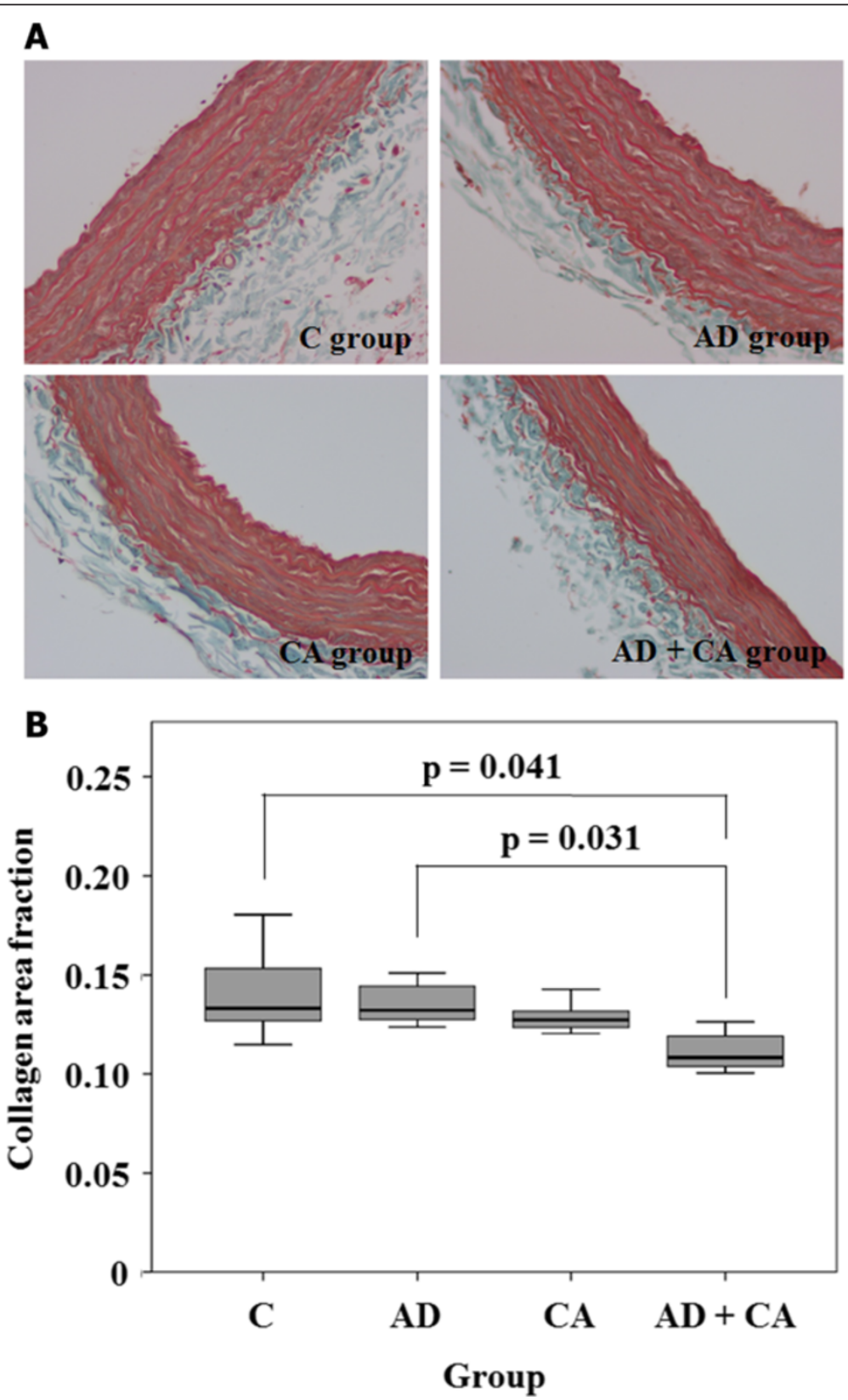

Figure 4 Goldner's trichrome stained section and collagen area fraction. (A) Goldner's trichrome-stained section $(\times 400)$ in $C$ group, AD group, CA group, and AD + CA group. (B) Collagen area fraction within the aorta in each group. C, control; AD, adriamycin-treated; CA, candesartan-treated; $A D+C A$, adriamycin + candesartan-treated. $p$ values were corrected by Dunnett method. 
the $\mathrm{CA}+\mathrm{AD}$ group, respectively. The $\mathrm{AD}$ group had a tendency of lower elastin area fraction than in the $C$ group $\left(p_{\text {Dun }}=0.086\right)$. And the elastic area fraction in the AD + CA group was significantly lesser than that in the CA group $\left(p_{\text {Dun }}=0.018\right)($ Figure 3$)$. However, there were no significant differences in the elastin area fraction between the $\mathrm{C}$ group and the $\mathrm{CA}$ group and between the $\mathrm{AD}$ group and the $\mathrm{AD}+\mathrm{CA}$ group. In Goldner's trichrome-stained section, the collagen area fraction were 0.15 (range, 0.13 to 0.19 ), 0.15 (range, 0.14 to 0.16 ), 0.14 (range, 0.14 to 0.15 ), and 0.12 (range, 0.11 to 0.14 ) in the $C$ group, the $\mathrm{AD}$ group, the $\mathrm{CA}$ group, and the $\mathrm{AD}+\mathrm{CA}$ group, respectively. The collagen area fraction in $\mathrm{AD}+\mathrm{CA}$ group was significantly lower than that in the $C$ group and the $\operatorname{AD}$ group ( $p_{\text {Dun }}=0.041$ and 0.031 , respectively) (Figure 4$)$. Collagen/elastin ratio were 0.97 (range, 0.77 to 2.24), 2.19 (range, 1.77 to 4.09 ), 1.06 (range, 0.82 to 1.46), and 1.54 (range, 1.20 to 2.08 ) in the $\mathrm{C}$ group, the $\mathrm{AD}$ group, the $\mathrm{CA}$ group, and the $\mathrm{CA}+\mathrm{AD}$ group, respectively. There were no significant differences in collagen/elastin ratio between the $\mathrm{C}$ group and the $\mathrm{AD}$ group $(p=0.093)$, between the $C$ group and the CA group $(p=0.589)$, between the $\mathrm{AD}$ group and the $\mathrm{AD}+\mathrm{CA}$ group $(p=0.240)$, and between the $\mathrm{CA}$ group and the $\mathrm{AD}+\mathrm{CA}$ group $(p=0.180)$ (Figure 5). In electron microscopic examination of the endothelial cells, we observed intact endothelial layers of the aorta in nine rats (three rats of the $\mathrm{C}$ group, one rat of the $\mathrm{AD}$ group, three rats of the $\mathrm{CA}$ group, and two rats of the $\mathrm{AD}+\mathrm{CA}$ group). There were no significant findings in the endothelial cells in the electron microscopic examination. In electron microscopic examination of the endothelial layers, there were

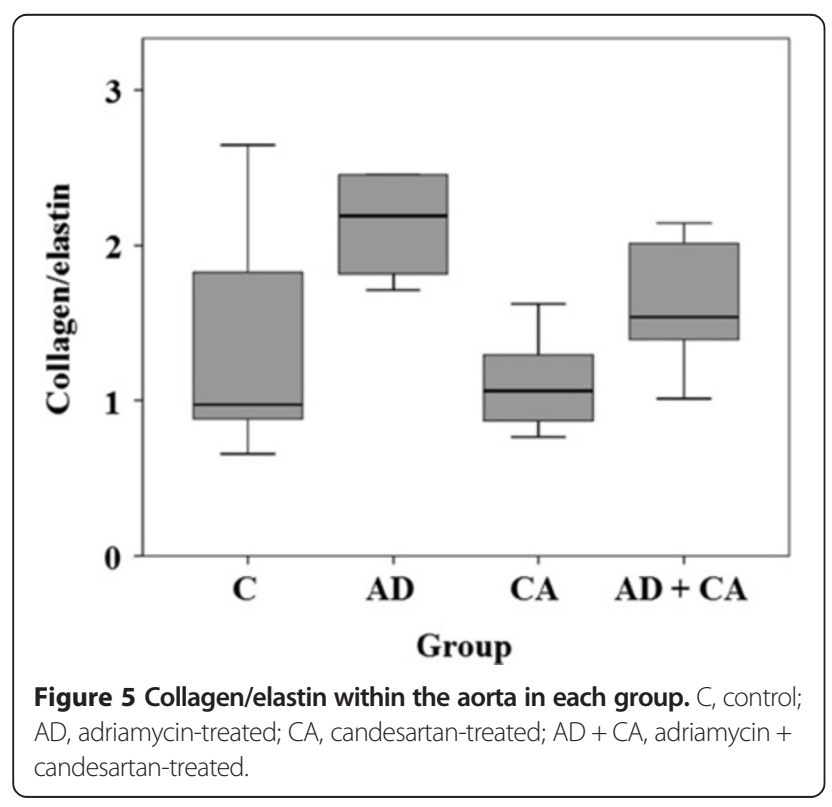

cytoplasmic vacuoles and degeneration of nuclei in a rat in the AD group (Figure 6). No specific changes were detected in the other groups.

\section{Discussion}

This pilot study demonstrated that the adriamycintreated groups had a tendency of lower elastin area fraction than the adriamycin-untreated groups. And candesartan decreased the thickness of tunica media in the aorta in adriamycin-treated and adriamycin-untreated rats and the quantity of collagen fibers in the aorta of adriamycin-treated rats. According to the results of the present study, adriamycin had a tendency of reduced quantity of elastin fibers in the aorta. As a result, elastance of the aorta could decrease and aortic stiffness could increase [9]. Decreased elastance and increased stiffness of the aorta mean pathologic processes and structural remodeling of the aorta. It could aggravate hypertension and cause target organ damage including left ventricular hypertrophy $[10,11]$. Decrement of aortic elastance could result in aggravation of adriamycininduced cardiotoxicity by myocardial fibrosis and structural remodeling. In addition, candesartan decreased the tunica media thickness and the quantity of collagen fibers in the aorta in the present study. It was because, generally, angiotensin receptor blockers have the effects of mitigating fibrosis by decreasing thickness of tunica media and collagenogenesis. Reduction of the quantity of collagen fibers means mitigation of fibrosis and reverse of structural remodeling of the aorta. Decrease of tunica media thickness could be relative to reverse of structural remodeling of the aorta. However, candesartan could not show the mitigation of decrement of elastin fibers by adriamycin.

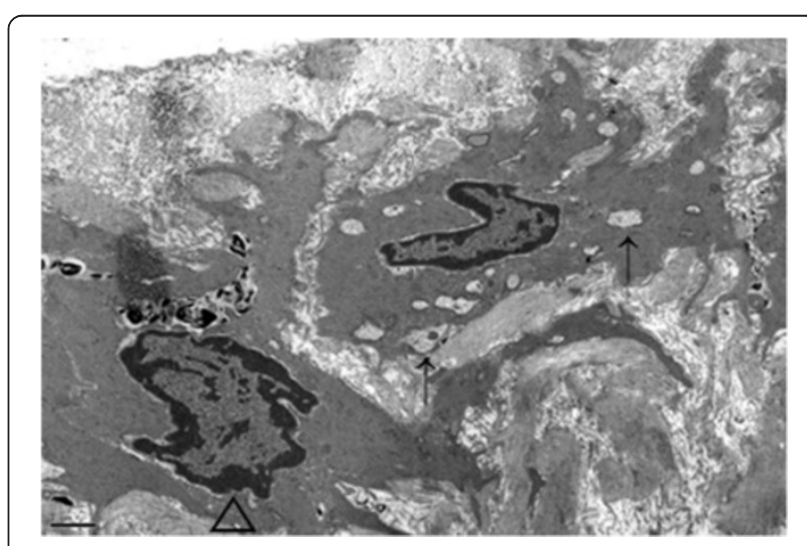

Figure 6 Cytoplasmic vacuoles (arrow) and degenerative nucleus (arrow head) in the endothelial cells in the $A D$ group. Electron microscopy, $\times 8,000$. AD, adriamycin-treated. 
Because fibrosis plays an important role in cardiomyopathy and vasculopathy, drugs that have an effect on the fibrotic process are essential for the treatment of cardiomyopathy and vasculopathy. Angiotensin converting enzyme inhibitors or angiotensin receptor blockers can regulate the renin-angiotensin-aldosterone system and ameliorate the fibrotic process [6]. In addition, it was reported that spironolactone, an aldosterone receptor blocker, and torasemide, a loop diuretic, can reduce fibrosis $[12,13]$.

Because the aorta is directly connected to the heart, the heart and aorta often share the same pathophysiologic processes. It has been reported that adriamycin induces aortic toxicity [14]. However, the mechanisms of adriamycininduced aortopathy are not known in detail. The suggested mechanisms of adriamycin-induced vasculopathy were endothelial dysfunction [15-18], suppression of endothelin-1 in endothelial cells [19], degeneration of endothelial structures [14], apoptosis of the endothelial cells [20], enhancement of procoagulant activity [21], smooth muscle dysfunction, and dysregulation of its calcium contents $[10,22]$. In addition, adriamycin could induce not only cardiovascular toxicity but also nephrotoxicity by alteration of glomerular endothelial cells [16,23].

Usually, injection of adriamycin induces retardation of weight gain [7]. In this study, adriamycin- or candesartantreated rats reduced weight gain compared with rats in control group, although there was no statistical significance. In adriamycin-treated rats, it could be because adriamycin has systemic toxicity. It has reported that incidence of gastrointestinal adverse effects of candesartan was $1 \%$ in human study [24]. In adriamycin-treated rats, reduced weight gain might be due to gastrointestinal adverse effects of candesartan.

We have shown in the previous report that collagen in the myocardium significantly increased in adriamycininduced cardiomyopathy rat model [7]. However, collagen area fraction between the $\mathrm{C}$ group and the $\mathrm{AD}$ group was not significantly different in the present study. It could be because the mechanisms of aortopathy could be different from those of cardiomyopathy and because the numbers of rats and the duration from the drug administration to sacrifice could be short.

In this study, ultramicroscopic structural changes were not obvious. It might be because the adriamycin-induced microscopic changes in the aorta were slower than those in cardiomyopathy due to the extracellular matrix of the aorta. It might be because the rats in the present study were too young to present the microscopic pathologic processes including atherosclerotic changes.

We had some limitations of the present study. We could not confirm the development of cardiomyopathy because we did not perform echocardiography in the rats. We could not show microscopic and ultramicroscopic changes of endothelial cells by adriamycin and candesartan because the endothelial layers were damaged in the processes of fixation and staining.

\section{Conclusions}

Adriamycin had a tendency of decreasing the quantity of elastin fibers, and candesartan cannot mitigate the effects of adriamycin on elastin fibers. In oncology clinic, physicians should be cautious about not only adriamycininduced cardiotoxicity but also adriamycin-induced vascular changes. Further studies are necessary to evaluate the effects of angiotensin-converting enzyme inhibitors or angiotensin receptor blockers on the adriamycin-induced vascular changes.

\section{Abbreviations}

AD: Adriamycin; C: Control; CA: Candesartan; $P_{\text {Dun: }}$ Dunnett-corrected $p$ value.

Competing interests

The authors declare that they have no competing interests.

\section{Authors' contributions}

JSU and WBC participated in the design of the study and performed data collection, statistical analysis, and interpretation and drafted the manuscript. JSY carried out animal care and microscopic studies and performed data collection. YSO performed and participated in the statistical analysis and interpretation and helped to draft the manuscript. HJY conceived of the study and participated in its design and coordination and helped to draft the manuscript. All authors read and approved the final manuscript.

\section{Acknowledgements}

This work was supported by the funds of Korean Society of Hypertension and Research Foundation of Internal Medicine, The Catholic University of Korea in 2010 .

\section{Author details}

${ }^{1}$ Department of Cardiology, Severance Hospital, Yonsei University College of Medicine, Seoul, Korea. ${ }^{2}$ Department of Cardiology, Catholic University of Korea College of Medicine, Seoul, Korea. ${ }^{3}$ Clinical Research Center, Yeouido St Mary's Hospital, Seoul, Korea.

Received: 27 March 2014 Accepted: 10 September 2014 Published: 25 September 2014

\section{References}

1. Weiss RB: The anthracyclines: will we ever find a better doxorubicin? Semin Oncol 1992, 19:670-86.

2. Chatterjee K, Zhang J, Honbo N, Karliner JS: Doxorubicin cardiomyopathy. Cardiology 2010, 115:155-62.

3. Murata T, Yamawaki H, Hori M, Sato K, Ozaki H, Karaki H: Chronic vascular toxicity of doxorubicin in an organ-cultured artery. Br J Pharmacol 2001, 132:1365-73.

4. Murata T, Yamawaki H, Yoshimoto R, Hori M, Sato K, Ozaki H, Karahi H: Chronic effect of doxorubicin on vascular endothelium assessed by organ culture study. Life Sci 2001, 69:2685-95.

5. Wu S, Ko YS, Teng MS, Ko YL, Hsu LA, Hsueh C, Chou YY, Liew CC, Lee YS: Adriamycin-induced cardiomyocyte and endothelial cell apoptosis: in vitro and in vivo studies. J Mol Cell Cardiol 2002, 34:1595-607.

6. Jugdutt BI: Pleiotropic effects of cardiac drugs on healing post-MI: the good, bad, and ugly. Heart Fail Rev 2008, 13:439-52.

7. Uhm JS, Youn HJ, Choi YS, Lee DH, Yun HJ, Park CS, Oh YS, Chung WS, Kim $\mathrm{JH}$, Choi KB, Hong SJ: Comparison of adriamycin-induced cardiomyopathy in normotensive rats and spontaneously hypertensive rats. Korean Hypertension J 2006, 12:23-30.

8. Konietschke F, Hothorn LA, Brunner E: Rank-based multiple test procedures and simultaneous confidence intervals. Electron J Stat 2012, 6:738-59. 
9. Wagenseil JE, Mecham RP: Elastin in large artery stiffness and hypertension. J Cardiovasc Transl Res 2012, 5:264-73.

10. De Wildt DJ, de Jong Y, Hillen FC, Steerenberg PA, van Hoesel QG: Cardiovascular effects of doxorubicin-induced toxicity in the intact Lou/M Wsl rat and in isolated heart preparations. J Pharmacol Exp Ther 1985, 235:234-40.

11. Kim TS, Youn HJ: From hypertension to heart failure. Korean Hypertension J 2009, 15:1-11.

12. Pitt B, Zannad F, Remme WJ, Cody R, Castaigne A, Perez A, Palensky J, Wittes J: The effect of spironolactone on morbidity and mortality in patients with severe heart failure. Randomized Aldactone Evaluation Study Investigators. N Engl J Med 1999, 341:709-17.

13. Lopez B, Querejeta R, Gonzalez A, Sanchez E, Larman M, Diez J: Effects of loop diuretics on myocardial fibrosis and collagen type I turnover in chronic heart failure. J Am Coll Cardiol 2004, 43:2028-35.

14. Yamaç D, Elmas C, Ozogul C, Keskil Z, Dursun A: Ultrastructural damage in vascular endothelium in rats treated with paclitaxel and doxorubicin. Ultrastruct Pathol 2006, 30:103-10.

15. Nicolson GL, Custead SE: Effects of chemotherapeutic drugs on platelet and metastatic tumor cell-endothelial cell interactions as a model for assessing vascular endothelial integrity. Cancer Res 1985, 45:331-6.

16. Ulu N, Buikema H, van Gilst WH, Navis G: Vascular dysfunction in adriamycin nephrosis: different effects of adriamycin exposure and nephrosis. Nephrol Dial Transplant 2008, 23:1854-60.

17. Den Hartog GJ, Boots AW, Haenen GR, van der Vijgh WJ, Bast A: Lack of inhibition of endothelial nitric oxide synthase in the isolated rat aorta by doxorubicin. Toxicol In Vitro 2003, 17:165-7.

18. Vasquez-Vivar J, Martasek P, Hogg N, Masters BS, Pritchard KA Jr, Kalyanaraman B: Endothelial nitric oxide synthase-dependent superoxide generation from adriamycin. Biochemistry 1997, 36:11293-7.

19. Keltai K, Cervenak L, Mako V, Doleschall Z, Zsary A, Karadi I: Doxorubicin selectively suppresses mRNA expression and production of endothelin-1 in endothelial cells. Vascul Pharmacol 2010, 53:209-14.

20. Maney SK, Johnson AM, Sampath Kumar A, Nair V, Santhosh Kumar TR, Kartha CC: Effect of apoptosis-inducing antitumor agents on endocardial endothelial cells. Cardiovasc Toxicol 2011, 11:253-62.

21. Swystun LL, Shin LY, Beaudin S, Liaw PC: Chemotherapeutic agents doxorubicin and epirubicin induce a procoagulant phenotype on endothelial cells and blood monocytes. J Thromb Haemost 2009, 7:619-26.

22. Dalske HF, Hardy K: Effect of low-dose doxorubicin on calcium content and norepinephrine response in rat aorta. Eur J Cancer Clin Oncol 1988, 24:979-83.

23. Jeansson M, Bjorck K, Tenstad O, Haraldsson B: Adriamycin alters glomerular endothelium to induce proteinuria. J Am Soc Nephrol 2009, 20:114-22.

24. Julius S, Nesbitt SD, Egan BM, Weber MA, Michelson EL, Kaciroti N, Black HR, Grimm RH Jr, Messerli FH, Oparil S, Schork MA: Feasibility of treating prehypertension with an angiotensin-receptor blocker. N Engl J Med 2006, 354:1685-97.

doi:10.1186/2056-5909-1-2

Cite this article as: Uhm et al.: Effects of adriamycin and candesartan on the collagen and elastin of the aorta in rats. Clinical Hypertension 2014 1:2.

\section{Submit your next manuscript to BioMed Central and take full advantage of:}

- Convenient online submission

- Thorough peer review

- No space constraints or color figure charges

- Immediate publication on acceptance

- Inclusion in PubMed, CAS, Scopus and Google Scholar

- Research which is freely available for redistribution 